\title{
Mucormicosis rinosinusal por Mucor hiemalis en una paciente con leucemia mieloide aguda
}

\author{
(Rhinosinusal mucormycosis by Mucor hiemalis in a patient with acute \\ myeloid leukemia)
}

Mariajosé García $\mathbf{S}^{1 *}$, Verónica Contreras $\mathbf{T}^{\mathbf{1}}$, Bernardita Rojas $\mathbf{M}^{2}$, Rodrigo Cruz Choappa ${ }^{3}$

1. Becada Medicina Interna Hospital Carlos van Buren, Valparaíso. 2. Hematóloga. Hospital Carlos van Buren, Valparaíso. 3. Laboratorio de Micología Universidad de Valparaíso *Autora para correspondencia: Mjgarcia.s84@gmail.com

RECIBIDO:11 de Noviembre de 2016 APROBADO:23 de Noviembre de 2016

DOI: 10.22370/bolmicol.2016.31.2.493

Palabras clave: Leucemia; mucormicosis; neutropenia febril

Key words: Leukemia; mucormycosis; febrile neutropenia

\section{RESUMEN}

Se reporta un caso clínico de una paciente femenina de 41 años, con antecedentes de leucemia mieloide aguda (LMA) en remisión. Estudiada por hematología, se confirmó recaída de LMA M4. Se inició quimioterapia. La paciente evolucionó con pancitopenia severa. Presentó dos episodios de neutropenia febril, el primero fue asociado a un absceso glúteo que se trató con antibacterianos, y el segundo a compromiso rinosinusal y úlcera necrótica de punta nasal, columela, tabique, cornete inferior izquierdo y paladar duro. Debido a la clínica e imágenes radiológicas, se sospechó mucormicosis, por lo que se realizó cirugía con debridación extensa y se inició tratamiento antimicótico con anfotericina $\mathrm{B}$ desoxicolato. El cultivo de tejido informó abundante desarrollo de Mucor hiemalis. Se mantuvo pancitopénica durante aproximadamente un mes, siendo diariamente evaluada por un equipo multidisciplinario. Se hicieron varios aseos quirúrgicos, en el último se encontró tejido vital. La paciente completó diez días con anfotericina B desoxicolato y posteriormente se hizo traslape a posaconazol oral. Se realizó mielograma de control que evidenció remisión completa de recaída de LMA. Se dio de alta a su domicilio al día 40 de hospitalización, con hemograma adecuado y tratamiento oral con posaconazol para completar 6 semanas en total.

\section{ABSTRACT}

We report a case of a 41-years-old female patient with a history of acute myeloid leukemia (AML) in remission. Hematology studies confirmed relapse of AML M4. Chemotherapy was started. The patient developed severe pancytopenia. She presented two episodes of febrile neutropenia, 
the first one was associated with a gluteal abscess that was treated with antibacterials, and the second to rhinosinusal involvement and necrotic ulcer of nasal tip, columella, septum, left inferior turbinate and hard palate. Due to clinical and radiological imaging, mucormycosis was suspected, so surgery was performed with extensive debridement and antifungal treatment with amphotericin B deoxicholate was initiated. Tissue culture reported abundant development of Mucor hiemalis. She remained pancytopenic for approximately one month, being evaluated daily by a multidisciplinary team. Several surgical were made, finding vital tissue in the last perform. The patient completed ten days with amphotericin B deoxicholate and later was overlapped to oral posaconazole. A control myelogram was performed, showing complete remission of AML. She was discharged to her home at day 40 of hospitalization, with adequate blood count and oral treatment with posaconazole to complete 6 weeks in total.

\section{INTRODUCCIÓN}

Las infecciones invasoras por hongos filamentosos son una causa importante de morbilidad y mortalidad en pacientes inmunocomprometidos con enfermedades hematológicas malignas. $\mathrm{Si}$ bien la aspergilosis invasora sigue siendo la mas frecuente de estas infecciones, agentes pertenecientes al orden mucorales (mucormicosis) son una causa cada vez más frecuente y con mayor mortalidad asociada en muchos centros alrededor del mundo ${ }^{1}$. Generalmente es una infección aguda y de desarrollo rápido, siendo la enfermedad micótica de evolución más fulminante en el hombre ${ }^{2,3}$. Las especies aisladas con mayor frecuencia son Rhizopus oryzae, (comprendiendo mas de la mitad de los aislamientos), seguido de R. microsporus, Mucor circinelloides y Lichtheimia corymbifera ${ }^{2}$. Todas éstas son de distribución mundial y el reservorio principal son las materias orgánicas como la tierra y los restos vegetales ${ }^{4}$. Estos organismos viven en el ambiente y penetran en el organismo por inha- lación de esporas a través de la vía aérea; a través de la piel por inoculación directa de conidios ${ }^{1}$ (por heridas, catéteres, líneas venosas); o por vía gastrointestinal $1^{5,6}$.

El poder patógeno intrínseco de los mucorales es mínimo en individuos inmunocompetentes, pero en pacientes con alteración de la inmunidad pueden causar infecciones graves e incluso mortales de presentación fulminante ${ }^{4}$. Se pueden identificar como factores de riesgo para mucormicosis, la cetoacidosis diabética, leucemias y linfomas con neutropenias prolongadas, sindrome urémico, medicamentos que alteran la respuesta inmune (ej: corticoides, agentes citotóxicos), grandes quemados, desnutrición calórico proteica y prematurez $z^{4}$.

Existen pocos datos epidemiológicos sobre la mucormicosis. La incidencia se ha incrementado en las neoplasias hematológicas durante la última década. En un estudio reciente de Francia, la tasa de incidencia anual de mucormicosis en pacientes con neoplasia hematológica aumentó con el tiempo de 0,7 a 1,2 casos / millón de personas desde 1997 a 2006 ( $+24 \%$ por año). Antes de esto, la mucormicosis se diagnosticaba a menudo en las autopsias y su incidencia en estos estudios estaba entre el 0,4\% y el $0,9 \%$ en los pacientes con neoplasia maligna. Entre los pacientes sometidos a tratamientos convencionales, los con LMA se encuentran en mayor riesgo, con tasas de incidencia que oscilan entre el $1 \%$ y el $1,9 \%$ en diferentes series. Por el contrario, la mucormicosis es rara en otras neoplasias hematológicas agudas o crónicas $(0,1 \%)^{1}$.

Los mucorales son hongos con gran capacidad para invadir los vasos sanguíneos y, a través de ellos, diseminarse rápidamente por órganos y estructuras adyacentes. Esto es la principal causa de la alta tasa de mortalidad ${ }^{7}$.

Todas las especies de mucorales producen abundantes esporas que son fácilmente difundidas mediante aerosoles, mecanismo por el que estos hongos se dispersan causando colonizaciones o infecciones rinosinusales o pulmonares en los huéspedes expuestos y en riesgo. Aunque las esporangiosporas son las formas infecciosas típicas de 
la mucormicosis, las hifas angioinvasoras son las causantes de la invasión tisular y de la diseminación en condiciones favorables de $\mathrm{pH}$, concentración de hierro y defectos en la inmunidad celular ${ }^{2}$.

Los sitios que con mayor frecuencia se afectan son los senos paranasales (SPN) (39\%), los pulmones $(24 \%)$ y la piel $(19 \%)^{8}$. Otros sitios incluyen, el cerebro, el tracto digestivo o la enfermedad diseminada con más de un sitio afectado ${ }^{1}$.

Aproximadamente, la mortalidad global de los pacientes con mucormicosis es del $40 \%$, aunque en pacientes hematológicos supera el $60 \%$. La tasa de supervivencia varía según la especie causal, la enfermedad de base, la extensión de la enfermedad y el tipo y momento de la cirugía. Además, la supervivencia está relacionada con la forma clínica de presentación: $90 \%$ en las formas cutáneas aisladas, $87 \%$ en sinusitis sin afectación cerebral, $45 \%$ en formas rinocerebrales, $36 \%$ en las pulmonares, $33 \%$ en la enfermedad cerebral focal, $16 \%$ en las formas diseminadas y $10 \%$ en las gastrointestinales ${ }^{9,10}$.

Debido a la gran velocidad de crecimiento y capacidad invasora de estos hongos, el diagnóstico precoz de la mucormicosis es clave para asegurar el éxito terapéutico ${ }^{10}$.La terapia incluye farmacos antifúngicos y la intervención quirúrgica con debridación amplia, y resección de los tejidos $\operatorname{afectados}^{4,11}$.

El objetivo de este trabajo es presentar un caso de mucormicosis rinosinusal por M. hiemalis, explicar los factores de riesgo, diagnostico y tratamiento.

\section{CASO CLÍNICO}

Paciente mujer de 41 años, con antecedentes de Hipotiroidismo, LMA M4 con citogenética de buen pronóstico, diagnosticada en junio del 2015, que debutó con leucocitosis $157.000 \mathrm{~mm}$, $71 \%$ blastos en sangre periférica y trombocitopenia. Completó terapia de inducción y consolidación, entre junio y octubre del 2015, con remisión completa.
En el seguimiento ambulatorio en el policlínico de hematología, presentó recaída en Julio del 2016 (en el hemograma destacó, 17\% blastos y trombocitopenia de $76.000 \mathrm{~mm} 3$ ).

Se diagnosticó LMA M4 en recaída a un año de remisión. Se hospitalizó en unidad de cuidados intermedios (UCIM) del Hospital Carlos van Buren el 25 de Julio para realizar quimioterapia de recaída, la que se inició el 27 de Julio. Evolucionó con pancitopenia y neutropenia profunda y prolongada (durante casi un mes), pese a dosis doble de factor estimulante de colonias y sensibilización a plaquetas. Fue politransfundida.

Presentó neutropenia febril (5 de agosto), de los exámenes destacó 300 glóbulos blancos mm3, elevación de índice de actividad inflamatoria, se pancultivó y se inició tratamiento empírico con cefepime 1 gramo cada 8 horas.

La paciente presentó odontalgia y leve dolor nasal, por lo que se realizó una tomografía axial computada (TAC) de SPN el 7 de agosto, la que mostró compromiso inflamatorio inespecífico etmoidal izquierdo y maxilar bilateral (Figuras 1 y 2). Simultáneamente a esto presentó un absceso glúteo, por lo que se agregó vancomicina ev 1 gramo cada 12 horas. Completó 7 días de tratamiento antibiótico, con regresión completa del absceso glúteo, disminución de la PCR, persistencia del dolor nasal, sin nuevos episodios de fiebre.

El 15 de agosto, evolucionó con franco dolor nasal y ulcera necrótica de punta nasal, columela, tabique, cornete inferior izquierdo y paladar duro. Se realizó un nuevo TAC que mostró extensa infiltración nasal, engrosamiento del vestíbulo y partes blandas a nivel de cornete inferior izquierdo y tabique nasal (Figuras 3 y 4). Se planteó probable mucormicosis rinosinusal, se evaluó en conjunto con otorrinolaringólogo e infectólogo y se decidió realizar cirugía con debridación extensa e iniciar tratamiento con anfotericina $\mathrm{B}$ desoxicolato.

La biopsia de nariz fue informada con inclusiones virales epidérmicas de tipo herpes. Además de tejido hemorrágico con abundantes bacilos 
bacterianos, sin observar elementos micóticos. En el cultivo de tejido hubo abundante desarrollo de M. hiemalis (Figuras 5, 6 y 7).

La paciente evolucionó con lenta recuperación de la pancitopenia. Fue evaluada diariamente por hematólogos, otorrinolaringólogos, infectólogos y médicos de UCIM, sin evidenciar progresión de la lesión nasal. El último aseo quirúrgico se realizó el día 29 de agosto y se encontró tejido vital.

Se trató diez días con anfotericina B desoxicolato y posteriormente se hizo traslape a pozaconazol oral. Como reacción adversa a anfotericina, presentó falla renal aguda, hipokalemia y dermatitis descamativa generalizada. Se recuperó de la pancitopenia severa el día 22 de agosto y se realizó un mielograma el día 30 de agosto que evidenció remisión completa de recaída de LMA. Durante la hospitalización, se realizaron pruebas seriadas de galactomanano en sangre, resultando todas negativas.

Se dio de alta a su domicilio al día 40 de hospitalización, con hemograma adecuado (leucocitos de $19.000 \mathrm{~mm} 3,81 \%$ de neutrófilos, $0 \%$ de blastos, hematocrito de $22 \%$, hemoglobina de 7.7 $\mathrm{g} / \mathrm{dl}$ y recuento de plaquetas de $69.000 \mathrm{~mm} 3)$, y con tratamiento oral con posaconazol para comple$\operatorname{tar} 6$ semanas en total.

Hasta la fecha ha mantenido sus controles en policlínico de hematología en buenas condiciones generales, sin otras complicaciones.
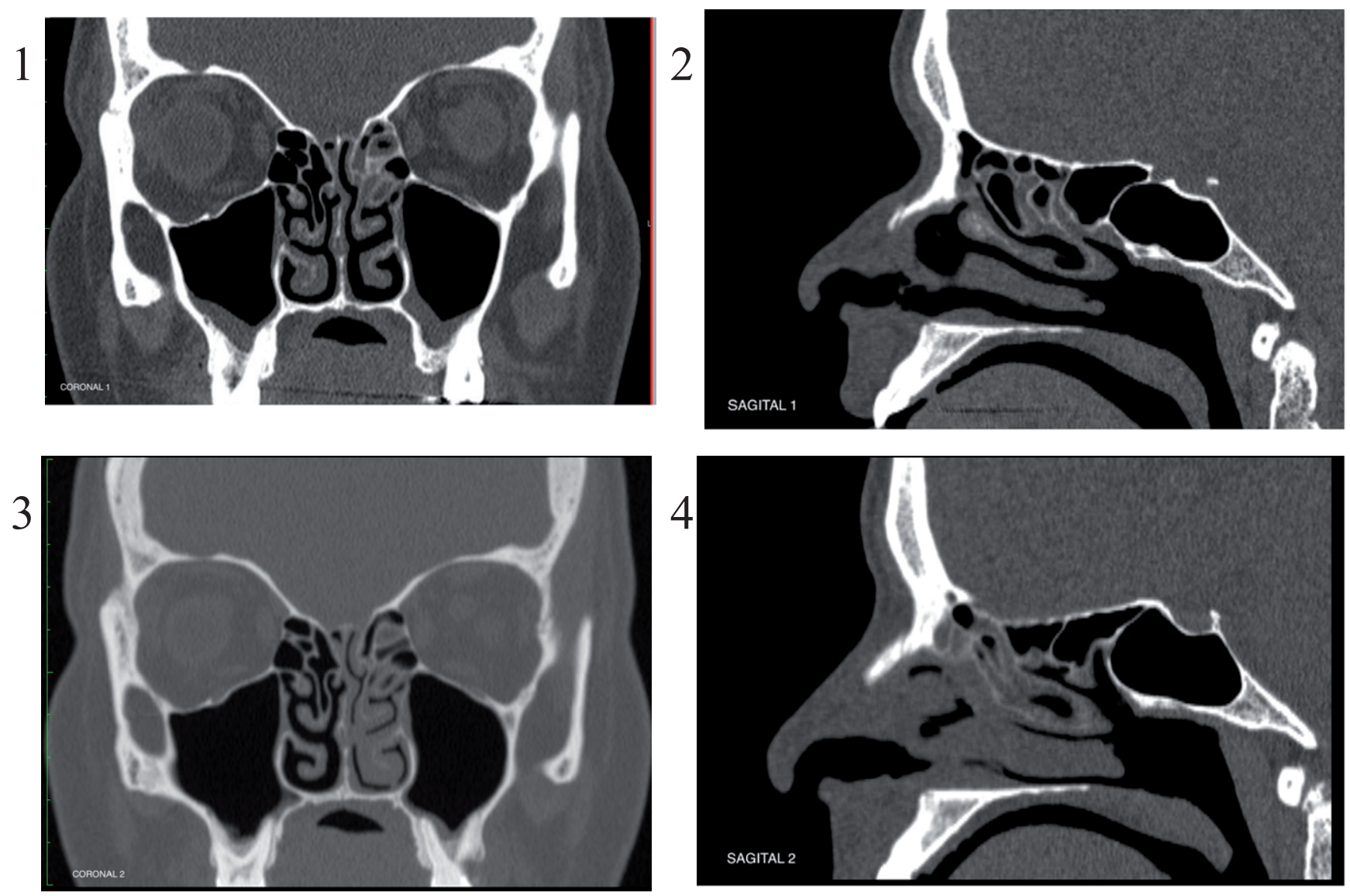

Figuras 1 y 2. En la imagen de TAC de senos paranasales se observa infiltración etmoidal izquierda y ocupación maxilar bilateral.

Figuras 3 y 4. En la imagen de TAC de senos paranasales se evidencia extensa infiltración nasal, engrosamiento del vestíbulo y partes blandas a nivel de cornete inferior izquierdo y tabique nasal. 

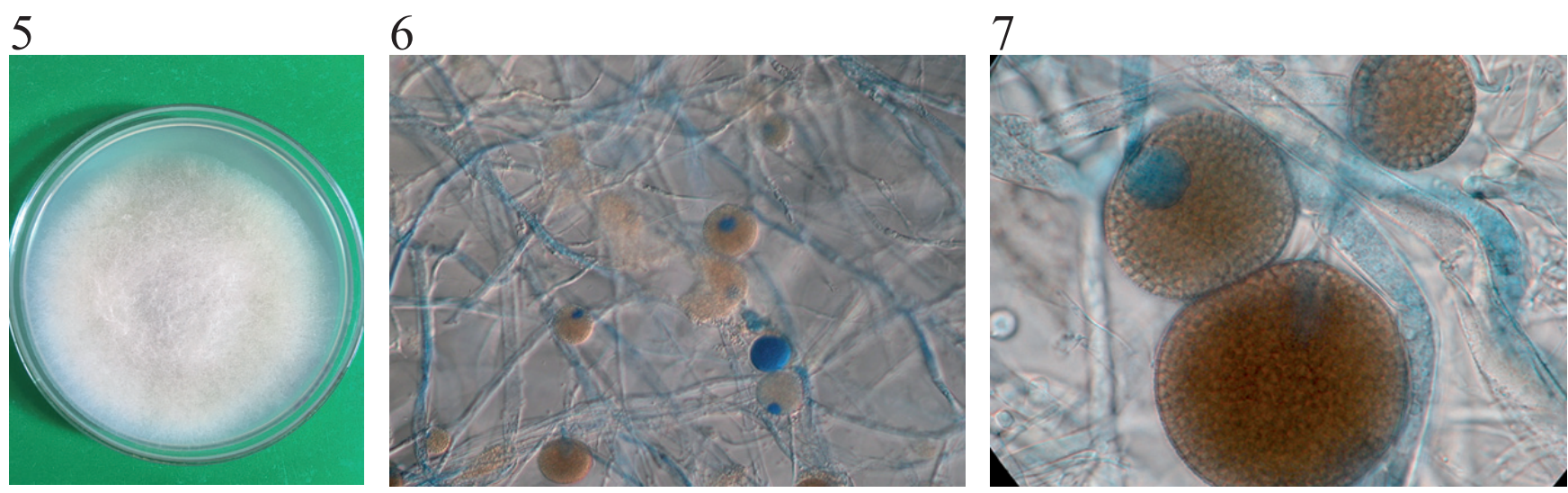

Figura 5. Colonia de rapido crecimiento a los 5 días de incubación.

Figura 6. Se observan esporangios con esporangioesporas en su interior. Aumento de 40X.

Figura 7. Se observan esporangios con esporangioesporas en su interior. Aumento de 100X.

\section{DISCUSIÓN}

Las mucormicosis son infecciones poco frecuentes pero graves, donde el inicio oportuno del tratamiento, puede prevenir la invasión y la muerte, es por esto la importancia del diagnóstico precoz. Para lograrlo, es importante tener una alta sospecha, reconocer los factores de riesgo del huésped, evaluar cuidadosamente las manifestaciones clínicas, usar precozmente las imágenes como TAC, la evaluación histológica y el uso óptimo de los métodos de microbiología clínica ${ }^{10}$.

La úlcera necrótica con escara negruzca a nivel nasal es una lesión característica y en los pacientes con factores de riesgo, puede ser un marcador temprano de mucormicosis invasora ${ }^{12}$. Para valorar la extensión de la lesión a tejidos adyacentes es útil la TAC y la resonancia magnética ${ }^{10}$. Sea cual sea el sitio clínico inicial involucrado, se debe realizar una TAC de SPN y de tórax, además de imágenes del cerebro, sobre todo si hay signos y síntomas sugerentes. Esto es importante, ya que el enfoque terapéutico es diferente en el caso de lesiones cerebrales ${ }^{1}$. Nuestra paciente presentó los signos clínicos y radiológicos sugerentes de mucormicosis.

A pesar de la gran angioinvasividad que caracteriza a las infecciones por mucorales, los hemocultivos de los pacientes generalmente son negativos ${ }^{10}$.

Se debe hacer todo lo posible para obtener biopsias para histopatología y cultivo. Esto es a menudo difícil en pacientes con neoplasias hematológicas debido a la presencia de trombocitopenia grave ${ }^{13}$. El material extraído de biopsias debe manejarse con cuidado porque los mucorales son difíciles de cultivar ${ }^{1}$. No debe triturarse ni homogeneizarse la muestra, ya que en estos hongos, al carecer sus hifas de septos, se pierde viabilidad rapidamente ${ }^{10}$. En nuestra paciente se pudo obtener muestra para biopsia, cultivo y además se realizó aseo quirúrgico.

El diagnóstico de infección por mucorales exige criterios rigurosos debido a su ubicuidad y a su alto poder contaminante en otros cultivos ${ }^{6}$. $\mathrm{La}$ observación del tejido preparado con hidróxido de potasio $(\mathrm{KOH})$ al $20 \%$ para destruir elementos tisulares, mostrará presencia de hifas gruesas como cinta de 10-20 mm de diámetro no septadas, con ramificaciones en ángulo recto. Sin embargo, se requiere un observador entrenado ya que a pesar del uso de $\mathrm{KOH}$, la presencia de detritus epiteliales podrían dificultar la observación ${ }^{4,13}$. En el examen microscópico en tejido fijado pueden utilizarse diferentes tinciones, como ácido peryódico de Schiff o tinción con metenamina de plata de 
Gomori, ambas sustentadas en la presencia de quitina y polisacáridos en la pared fúngica. Desde el servicio de Anatomía Patológica de nuestro hospital no se informó presencia de elementos fúngicos en la biopsia, lo que podría explicarse por la ausencia de invasión en la muestra enviada a este laboratorio. Sin embargo hubo abundante desarrollo de M. hiemalis en todas las muestras sembradas, lo que sumado al cuadro clínico sugerente, permitió considerar esta infección como una mucormicosis y tratarla como tal.

El cultivo de hongos permite la identificación definitiva de la especie. Los mucorales son hongos filamentosos que crecen rápidamente ( 2 a 5 días) en la mayoría de los medios de cultivo ${ }^{4}$, tales como agar Sabouraud y agar de papa dextrosa ${ }^{11}$. La sensibilidad de los cultivos no es óptima, ya que sólo aproximadamente un tercio de todos los especímenes positivos microscópicamente revelan cultivos positivos ${ }^{11}$.

El elevado número de hallazgos negativos en cultivo con resultados positivos microscópicamente, se puede explicar por el difícil crecimiento de estos hongos no septados, lo que los hace fácilmente dañables durante la manipulación de la muestra ${ }^{11}$. En nuestra paciente, las cuatro muestras de tejido cultivados a $27^{\circ} \mathrm{C}$ tuvieron desarrollo de $M$. hiemalis, por lo que es poco probable que haya sido una contaminación.

Cuando los cultivos son negativos, la identificación molecular de las muestras de tejidos puede confirmar el diagnóstico histológico. Sin embargo, en la actualidad, no existe un método estandarizado disponible ${ }^{1}$.

A diferencia de otras enfermedades fúngicas invasoras, no se han encontrado biomarcadores útiles que ayuden al diagnóstico de la mucormicosis. Tanto el galactomanano como el B-glucano no son componentes de la pared celular de los mucorales, por lo que son indetectables en las muestras clínicas de los pacientes con estas infecciones ${ }^{10}$. Sin embargo, tanto la prueba de galactomanano como 1,3-B-D-glucano, ayudan a descartar la as- pergilosis invasora, el diagnóstico diferencial más frecuente, o infecciones mixtas por Aspergillus y Mucorales ${ }^{1}$.

La infección por $M$. hiemalis es rara y de baja virulencia, principalmente porque esta especie no logra crecer a $37^{\circ} \mathrm{C}$, siendo su temperatura óptima de crecimiento sólo de $32^{\circ} \mathrm{C}$, por lo que se ha asociado principalmente a infecciones cutáneas, donde la temperatura puede ser mas baja que la central ${ }^{14}$.

El abordaje terapéutico de la mucormicosis es multimodal. Incluye el inicio precoz de terapia antifúngica efectiva, el desbridamiento quirúrgico extenso y temprano de lesiones necróticas y el control de la condición subyacente cuando sea posible. En pacientes con neutropenia están indicados los factores estimulantes de colonias de granulocitos ${ }^{1}$.

El antifúngico de primera línea, corresponde a las formulaciones lipídicas de anfotericina B. Como antifúngico de segunda línea, intolerancia a fármaco de primera línea o terapia de rescate, está indicado el posaconazol, o las combinaciones de anfotericina B con equinocandinas o posaconazol. El inicio de la terapia temprana se asocia con una mejor sobrevida. Las dosis, así como la duración del tratamiento aún no se han definido. Se usan dosis entre $5-10 \mathrm{mg} / \mathrm{kg} /$ día. El período de tratamiento debe determinarse individualmente, pero la terapia suele continuar durante al menos 6-8 semanas ${ }^{1}$.

La angio-invasividad de los agentes de mucormicosis, resulta en la formación de trombosis extensa, infartos y necrosis que dificultan la penetración de agentes antifúngicos en el sitio de infección. El desbridamiento oportuno de todo el tejido desvitalizado reduce la masa de la infección y previene la extensión a estructuras adyacentes. Esto no siempre es factible en pacientes con neoplasias hematológicas, que a menudo tienen trombocitopenia. Las recomendaciones relativas a la cirugía en la mucormicosis varían según el sitio y la extensión de la enfermedad. En la enfermedad rino-órbito-cerebral, el desbridamiento quirúrgico rápido, repetido si es necesario, se considera un 
componente crucial de la terapia exitosa ${ }^{1}$.

Se ha demostrado en estudios que la tasa de mortalidad de los pacientes que recibieron cirugía además de la terapia antifúngica es menor en comparación con los que recibieron antifúngicos sólos ${ }^{1}$. Nuestra paciente, recibió terapia antifúngica, desbridación precoz y factor estimulante de colonias de granulocitos.

Hasta hoy el diagnóstico precoz de las mucormicosis sigue siendo un reto para los médicos, por lo que sistemas de amplificación molecular avanzada, ensayos de detección de antígeno, perfiles proteómicos, $\mathrm{y}$ detección de metabolitos podrían complementar los enfoques existentes para mejorar la tasa de diagnóstico temprano de la mucormicosis invasora ${ }^{15}$. Actualmente se están evaluando métodos basados en técnicas de amplificación mediante PCR en tiempo real y de hibridación in situ empleando sondas de ADN con resultados esperanzadores ${ }^{16}$.

\section{REFERENCIAS}

1. Skiada A, Lanternier F, Groll AH, Pagano $\mathbf{L}$, Zimmerli S, Herbrecht $\mathbf{R}$ et al. Diagnosis and treatment of mucormycosis in patients with hematological malignancies: guidelines from the 3rd European Conference on Infections in Leukemia (ECIL 3). Haematologica. 2013 Apr; 98(4):492-504. Disponible en: http://www.haematologica.org/content/98/4/492

2. Petrikkos G. Skiada A. Lortholary O. Roilides E, Walsh TJ, Kontoyiannis DP. Epidemiology and Clinical Manifestations of $\mathrm{Mu}-$ cormycosis . Clin Infect Dis. (2012) 54 (suppl 1):S23-S34. Disponible en: http://cid.oxfordjournals.org/content/54/supp1_1/S23.long

\section{Fernández E. Dünner M. Mandiola C, Sil-} va M. Experiencia en mucormicosis Hospital Clínico Universidad de Chile. Revista Hospital Clínico Universidad de Chile Vol. $16 \mathrm{~N}^{\circ} 3$ año 2005. Disponible en: https://www.redclinica.cl/ Portals/0/Users/014/14/14/mucormicosis $\% 20$ 3-2005.pdf

4. García P. Beltrán C. Guzmán AM, León $\mathbf{P}$, Arredondo M, Fonseca $\mathbf{X}$. Diagnóstico rápido de dos casos de mucormicosis con tinción de blanco de calcoflúor. Rev. chil. infectol. v.18 n.4 Santiago 2001. Disponible en: http://
WwW.scielo.cl/scielo.php?script $=$ sci_arttext\&pi $\mathrm{d}=\mathrm{S} 0716-10182001000400007$

5. Silva P. Avilés C. Mucormicosis pulmonar. Rev. chil. infectol. 2004, vol.21, n.1, pp.61-64. Disponible en: http:// www.scielo.cl/scielo.php? pid=S $0716-$ $10182004000100009 \&$ script $=$ sci_abstract

6. Santos-Juanes J. Galache C. Pérez JA, Vásquez L, Cañizo C, Morán M. Mucormicosis diseminada en una paciente con leucemia mieloblástica aguda. Actas Dermosifiliogr 2001;92:154-157. Disponible en: www.elsevier. es/es-revista-semergen-medicina-familia-40pdf-12003939-S300

7. Malani AN, Kauffman CA. Changing epidemiology of rare mould infections: Implications for therapy. Drugs. 2007;67:1803-12. Disponible en: http://link.springer.com/article/10.2165/00003495-200767130-00001

8. Lanternier F, Sun H-Y, Ribaud P, Singh N, Kontoyiannis DP, Lortholary O. Mucormycosis in organ and stem cell transplant recipients. Clin. Infect. Dis. 54, 1-8 (2012). Disponible en: http://cid.oxfordjournals.org/content/54/11/1. long 
9. Gil-Lamaignere C, Simitsopoulou M. Roilides E, Maloukou A, Winn R, Walsh TJ. Interferon- $\gamma$ and granulocyte- macrophage colony-stimulating factor augment the activity of polymorphonuclear leukocytes against medica1ly important zygomycetes. J. Infect. Dis. (2005) 191 (7): 1180-1187. Disponible en: http://jid. oxfordjournals.org/content/191/7/1180.long

10. Pemán J, Salavert M. Enfermedad fúngica invasora por Scedosporium, Fusarium y $\mathrm{Mu}$ cor. Rev Iberoam Micol. 2014;31(4):242-248. Disponible en: http://linkinghub.elsevier.com/ retrieve/pii/S113014061400045X

11. Lackner M, Caramalho R, Lass-Flörl C. Review. Laboratory diagnosis of mucormycosis: current status \& future perspectives . Future Microbiol. (2014) 9(5), 683-695. Disponible en: http:// www.futuremedicine.com/doi/10.2217/fmb.14.23

12. Walsh T, Skiada A. Cornely O, Roilides E, Ibrahim A, Zaoutis T et al. Development of new strategies for early diagnosis of mucormycosis from bench to bedside. Mycoses, 2014, 57 (Suppl. 3), 2-7. Disponible en: http://onlinelibrary.wiley. com/doi/10.1111/myc.12249/abstract
13. Lass-Flörl C. Zygomycosis: conventional laboratory diagnosis. Clin Microbiol Infect. 2009;15 (Suppl 5):60-5. Disponible en: http:// www.clinicalmicrobiologyandinfection.com/article/S1198-743X(14)60731-X/abstract

14. Ribes JA, Vanover-Sams CL, Baker DJ. Zygomycetes in Human Disease. Clinical. Microbiology. Reviews. 2000; 13(2): 236301. Disponible en: http://cmr.asm.org/content/13/2/236.short

15. Walsh T. Gamaletsou M, McGinnis MR, Hayden RT, Kontoyiannis DP. Early Clinical and Laboratory Diagnosis of Invasive Pulmonary, Extrapulmonary, and Disseminated $\mathrm{Mu}$ cormycosis (Zygomycosis). Clin Infect Dis. (2012) 54 (suppl 1):S55-S60. Disponible en: http://cid.oxfordjournals.org/content/54/suppl_1/S55.full

16. Bernal-Martínez L, Buitrago MJ. Development of a single tube multiplex real-time PCR to detect the most clinically relevant Mucormycetes species. Clin Microbiol Infect. 2013;19:E17. Disponible en: http://www.sciencedirect. com/science/article/pii/S1 198743X14603190 